\title{
SYNTHESIS AND ANTITUBERCULAR ACTIVITY OF PYRIDAZINONE DERIVATIVES
}

\author{
ASIF HUSAIN ${ }^{*}$, AFTAB AHMAD ${ }^{2}$, ANIL BHANDARI', VEERMA RAM² \\ ${ }^{I}$ Department of Pharmaceutical Chemistry, Faculty of Pharmacy, Hamdard University, New Delhi-110062, India; ${ }^{2}$ Faculty of Pharmaceutical Sciences, \\ Jodhpur National University, Jodhpur, Rajasthan-342001, India
}

(Received: January 24, 2011 - Accepted: June 26, 2011)

\begin{abstract}
Two series of pyridazinone derivatives (19-34) were synthesized and evaluated for antitubercular activities against Mycobacterium tuberculosis $\mathrm{H}_{37} \mathrm{Rv}$ strain. The results illustrated that among the synthesized compounds, compound 25, 5-(4-hydroxy-3-methoxybenzyl)-3-(4-chloro-phenyl)-1,6-dihydro-6-pyridazinone emerged as a lead compound with good antitubercular activity. Four more compounds, (21, 22, 29 \& 33) were significant in their antitubercular action.
\end{abstract}

Key words: Pyridazinone, antitubercular, mycobacteria, furanone.

\section{INTRODUCTION}

During recent years pyridazinones have been a subject of intensive research owing to their wide spectrum of pharmacological activities. Differently substituted pyridazinones have been found to have potential antibacterial, antifungal and antiviral including anti-HIV activities [1-4]. Various 3-(2H)-pyridazinone derivatives have shown anticancer [4], analgesic $\&$ anti-inflammatory [4-6], anticonvulsant [7], cardiotonic \& hypotensive [8,9] and antiulcer activities [10].

Resistance of Mycobacterium tuberculosis strains to antitubercular agents is an increasing problem worldwide. However, potent new antimycobacterial drugs with new mechanism of action have not been developed in the last forty years [11]. TB is considered by the WHO to be the most important chronic communicable disease in the world. About $32 \%$ of the world's population is currently infected with TB. The emergence of AIDS, decline of socioeconomic standards and a reduced emphasis on tuberculosis control programs contribute to the disease's resurgence in industrialized countries [12]. If the present trend continues, tuberculosis is likely to claim more than 30 million lives within the new decade.

Now research effort towards the development of novel antitubercular agents is in the direction of discovering new classes of compounds, which are structurally different from known anti-tubercular drugs $[13,14]$. The current work describes the synthesis of newer $2(3 \mathrm{H})$-pyridazinones with encouraging antitubercular activity.

\section{EXPERIMENTAL}

\section{Chemistry}

Melting points were determined in open capillary tubes and are uncorrected. Thin-layer chromatography was carried out to monitor the reactions using silica gel $\mathrm{G}$ plates. The IR spectra were recorded in potassium bromide pellets using a Perkin-Elmer 1725X spectrophotometer. Elemental analyses were performed on a Perkin-Elmer 240 analyzer and the values were in range of $\pm 0.4 \%$ theoretical value for the element analyzed $(\mathrm{C}, \mathrm{H}, \mathrm{N}) .{ }^{1} \mathrm{H}-\mathrm{NMR}$ spectra were recorded on Bruker spectropsin DPX-300 $\mathrm{MHz}$ in $\mathrm{CDCl}_{3}$; chemical shift $(\delta)$ values are reported in parts per million $(\mathrm{ppm})$. The splitting pattern abbreviations are as follows: $s$, singlet; $d$, doublet; $m$, multiplet. Mass spectra under electron impact conditions (EI) were recorded at $70 \mathrm{eV}$ ionizing voltage with a VG Prospec instrument and are presented as $\mathrm{m} / \mathrm{z}$. Spectral data are consistent with the assigned structures.

Preparation of 3-(4-Chloro/methyl benzoyl)propionic acid $(1,2)$. The compounds, $\mathbf{1}$ and 2, were synthesized according to the reported method [14].

Preparation of 3-Arylidene-5-(4-chloro/methyl phenyl)-2(3H)-furanones (3-18). Compounds (3-18) were synthesized from 3-(4-chloro/methyl benozyl) propionic acid $(\mathbf{1 , 2})$ following literature method [14].

General Procedure for the synthesis of 5-(substituted benzyl)-3-aryl-1,6dihydro-6-pyridazinones (19-34). 2(3H)-Furanones (3-18) $(0.005 \mathrm{~mol})$ and hydrazine hydrate $(1-2 \mathrm{~mL})$ in $n$-propanol $(5-6 \mathrm{~mL})$ were refluxed for $3 \mathrm{~h}$.
After refluxing reaction mixture was poured onto crushed ice, a precipitate was obtained, which was filtered, dried and recrystallized from methanol to give TLC pure 5-(substituted benzyl)-3-aryl-1,6-dihydro-6-pyridazinone derivatives.

5-Benzyl-3-(4-chlorophenyl)-1,6-dihydro-6-pyridazinone (19): Yield: $59 \%$; m.p. $174{ }^{\circ} \mathrm{C}$; ${ }^{1} \mathrm{H}-\mathrm{NMR}\left(\mathrm{CDCl}_{3}, \delta\right.$, ppm): $3.78\left(\mathrm{~s}, 2 \mathrm{H}, \mathrm{CH}_{2}\right), 7.16(\mathrm{~s}, 1 \mathrm{H}$, $\mathrm{H}-4$, pyridazinone ring), 7.14-7.48 ( $\mathrm{m}, 5 \mathrm{H}$, benzyl ring), 7.42 and 7.73 (d, each, $J=8.1 \mathrm{~Hz}, 2 \mathrm{xA}_{2} \mathrm{~B}_{2}, p$-substituted phenyl ring), $10.72(\mathrm{~s}, 1 \mathrm{H}, \mathrm{NH})$; $\mathrm{MS}(\mathrm{m} / \mathrm{z})$ : 296( $\left.\mathrm{M}^{+}\right)$; IR $\left(\mathrm{cm}^{-1}, \mathrm{KBr}\right): 3186(\mathrm{NH}), 2949(\mathrm{CH}), 1683(\mathrm{CO}), 718$ (C-Cl); Anal calcd. for $\mathrm{C}_{17} \mathrm{H}_{13} \mathrm{ClN}_{2} \mathrm{O}: \mathrm{C}, 68.81 ; \mathrm{H}, 4.42 ; \mathrm{N}, 9.44$. Found: $\mathrm{C}, 68.93 ; \mathrm{H}, 4.45$; N, 9.43 .

5-(4-Chlorobenzyl)-3-(4-chlorophenyl)-1,6-dihydro-6-pyridazinone (20): Yield: $58 \%$; m.p. $196{ }^{\circ} \mathrm{C} ;{ }^{1} \mathrm{H}-\mathrm{NMR}\left(\mathrm{CDCl}_{3}, \delta\right.$, ppm): 3.95 (s, $\left.2 \mathrm{H}, \mathrm{CH}_{2}\right), 7.11$ (s, $1 \mathrm{H}, \mathrm{H}-4$, pyridazinone ring), $7.23(\mathrm{~m}, 4 \mathrm{H}, \mathrm{H}-2,3,5,6$, benzyl ring), 7.32 and 7.41 (d, each, $J=8.1 \mathrm{~Hz}, 2 \mathrm{xA}_{2} \mathrm{~B}_{2}, p$-substituted phenyl), 10.97 (s, $\left.1 \mathrm{H}, \mathrm{NH}\right)$; MS $(\mathrm{m} / \mathrm{z}): 330\left(\mathrm{M}^{+}\right)$; IR $\left(\mathrm{cm}^{-1}, \mathrm{KBr}\right): 3179(\mathrm{NH}), 2942(\mathrm{CH}), 1688(\mathrm{CO}), 726(\mathrm{C}-$ Cl); Anal calcd. for $\mathrm{C}_{17} \mathrm{H}_{12} \mathrm{Cl}_{2} \mathrm{~N}_{2} \mathrm{O}: \mathrm{C}, 61.65 ; \mathrm{H}, 3.65 ; \mathrm{N}, 8.46$. Found: $\mathrm{C}, 61.55$; $\mathrm{H}, 3.67 ; \mathrm{N}, 8.47$.

5-(4-Nitrobenzyl)-3-(4-chlorophenyl)-1,6-dihydro-6-pyridazinone (21): Yield: $53 \%$; m.p. $197^{\circ} \mathrm{C} ;{ }^{1} \mathrm{H}-\mathrm{NMR}\left(\mathrm{CDCl}_{3}, \delta\right.$, ppm): $3.71\left(\mathrm{~s}, 2 \mathrm{H}, \mathrm{CH}_{2}\right), 6.78$ (s, $1 \mathrm{H}, \mathrm{H}-4$, pyridazinone ring), $7.37(\mathrm{~m}, 4 \mathrm{H}, \mathrm{H}-2,3,5,6$, benzyl ring), 7.53 and 7.62 (d, each, $J=8.4 \mathrm{~Hz}, 2 \mathrm{xA}_{2} \mathrm{~B}_{2}, p$-substituted phenyl), 10.93 (s, 1H, NH); MS $(\mathrm{m} / \mathrm{z}): 341\left(\mathrm{M}^{+}\right)$; IR $\left(\mathrm{cm}^{-1}, \mathrm{KBr}\right): 3173(\mathrm{NH}), 2936(\mathrm{CH}), 1672(\mathrm{CO}), 707$ (C-Cl); Anal calcd. for $\mathrm{C}_{17} \mathrm{H}_{12} \mathrm{ClN}_{3} \mathrm{O}_{3}: \mathrm{C}, 59.75 ; \mathrm{H}, 3.54 ; \mathrm{N}, 12.30$. Found: $\mathrm{C}$, 59.63; H, 3.57; N, 12.31 .

5-(4-Hydroxybenzyl)-3-(4-chlorophenyl)-1,6-dihydro-6-pyridazinone (22): Yield: $57 \%$, m.p. $192{ }^{\circ} \mathrm{C}$; ${ }^{1} \mathrm{H}-\mathrm{NMR}\left(\mathrm{CDCl}_{3}, \delta, \mathrm{ppm}\right): 3.59(\mathrm{~s}, 2 \mathrm{H}$, $\left.\mathrm{CH}_{2}\right), 6.11(\mathrm{~m}, 1 \mathrm{H}, \mathrm{OH}), 7.06(\mathrm{~m}, 2 \mathrm{H}, \mathrm{H}-2,6$, benzyl ring), $7.31(\mathrm{~s}, 1 \mathrm{H}, \mathrm{H}-4$, pyridazinone ring), 7.47 and $7.71\left(\mathrm{~d}\right.$, each, $J=7.8 \mathrm{~Hz}, 2 \mathrm{xA}_{2} \mathrm{~B}_{2}, p$-substituted phenyl), 7.49 (m, 2H, H-3,5, benzyl ring), $9.41(\mathrm{~s}, 1 \mathrm{H}, \mathrm{NH}) ; \mathrm{MS}(\mathrm{m} / \mathrm{z}): 312\left(\mathrm{M}^{+}\right)$; IR ( $\left.\mathrm{cm}^{-1}, \mathrm{KBr}\right): 3178(\mathrm{NH}), 2942(\mathrm{CH}), 1686$ (CO), 722 (C-Cl); Anal calcd. for $\mathrm{C}_{17} \mathrm{H}_{13} \mathrm{ClN}_{2} \mathrm{O}_{2}: \mathrm{C}, 65.29 ; \mathrm{H}, 4.19 ; \mathrm{N}, 8.96$. Found: C, 65.39; H, 4.17; N, 8.97.

5-(4-Methylbenzyl)-3-(4-chlorophenyl)-1,6-dihydro-6-pyridazinone (23): Yield: $59 \%$; m.p. $186{ }^{\circ} \mathrm{C} ;{ }^{1} \mathrm{H}-\mathrm{NMR}\left(\mathrm{CDCl}_{3}, \delta\right.$, ppm): 2.27 (s, $\left.3 \mathrm{H}, \mathrm{CH}_{3}\right), 3.51$ $\left(\mathrm{s}, 2 \mathrm{H}, \mathrm{CH}_{2}\right), 6.60(\mathrm{~s}, 1 \mathrm{H}, \mathrm{H}-4$, pyridazinone ring), 7.13 and 7.36 (d, each, $J=7.8 \mathrm{~Hz}, 2 \mathrm{xA}_{2} \mathrm{~B}_{2}, p$-substituted benzyl ring), $7.34(\mathrm{~m}, 2 \mathrm{H}, \mathrm{H}-3,5$, phenyl ring), $7.61\left(\mathrm{~m}, 2 \mathrm{H}, \mathrm{H}-2,4\right.$, phenyl ring), $10.93(\mathrm{~s}, 1 \mathrm{H}, \mathrm{NH}) ; \mathrm{MS}(\mathrm{m} / \mathrm{z}): 310\left(\mathrm{M}^{+}\right)$; IR ( $\left.\mathrm{cm}^{-1}, \mathrm{KBr}\right): 3173(\mathrm{NH}), 2939(\mathrm{CH}), 1684(\mathrm{CO}), 708(\mathrm{C}-\mathrm{Cl})$; Anal calcd. for $\mathrm{C}_{18} \mathrm{H}_{15} \mathrm{ClN}_{2} \mathrm{O}: \mathrm{C}, 69.57 ; \mathrm{H}, 4.86 ; \mathrm{N}, 9.01$. Found: C, 69.53; H, 4.87; N, 9.03.

5-(4-Methoxybenzyl)-3-(4-chlorophenyl)-1,6-dihydro-6-pyridazinone (24): Yield: $61 \%$; m.p. $169{ }^{\circ} \mathrm{C}$; ${ }^{1} \mathrm{H}-\mathrm{NMR}\left(\mathrm{CDCl}_{3}, \delta\right.$, ppm): $3.42\left(\mathrm{~s}, 2 \mathrm{H}, \mathrm{CH}_{2}\right)$, $3.71\left(\mathrm{~s}, 3 \mathrm{H}, \mathrm{OCH}_{3}\right), 6.81(\mathrm{~s}, 1 \mathrm{H}, \mathrm{H}-4$, pyridazinone ring), 7.13 and $7.36(\mathrm{~d}$, each, $J=8.1 \mathrm{~Hz}, 2 \times \mathrm{A}_{2} \mathrm{~B}_{2}, p$-substituted benzyl ring), 7.59 (m, 2H, H-3,5, phenyl ring), $7.68\left(\mathrm{~m}, 2 \mathrm{H}, \mathrm{H}-2,4\right.$, phenyl ring), $10.73(\mathrm{~s}, 1 \mathrm{H}, \mathrm{NH})$; $\mathrm{MS}(\mathrm{m} / \mathrm{z}): 326\left(\mathrm{M}^{+}\right)$; IR ( $\left.\mathrm{cm}^{-1}, \mathrm{KBr}\right): 3167(\mathrm{NH}), 3002(\mathrm{CH}), 1675(\mathrm{CO}), 717(\mathrm{C}-\mathrm{Cl})$; Anal calcd. for $\mathrm{C}_{18} \mathrm{H}_{15} \mathrm{ClN}_{2} \mathrm{O}_{2}: \mathrm{C}, 66.16 ; \mathrm{H}, 4.63 ; \mathrm{N}, 8.57$. Found: $\mathrm{C}, 66.23 ; \mathrm{H}, 4.61 ; \mathrm{N}, 8.55$.

5-(4-Hydroxy-3-methoxybenzyl)-3-(4-chlorophenyl)-1,6-dihydro-6pyridazinone (25): Yield: $53 \%$; m.p. $191{ }^{\circ} \mathrm{C}$; ${ }^{1} \mathrm{H}-\mathrm{NMR}\left(\mathrm{CDCl}_{3}, \delta, \mathrm{ppm}\right): 3.48$ 
(s, $\left.2 \mathrm{H}, \mathrm{CH}_{2}\right), 3.64\left(\mathrm{~s}, 3 \mathrm{H}, \mathrm{OCH}_{3}\right), 6.53(\mathrm{~s}, 1 \mathrm{H}, \mathrm{H}-4$, pyridazinone ring), 7.077.29 (m, 3H, H-2,5,6, disubstituted benzyl ring), 7.46 and 7.71 (d, each, $J=8.1$ $\mathrm{Hz}, 2 \mathrm{xA}_{2} \mathrm{~B}_{2}, p$-substituted phenyl ring), $10.92(\mathrm{~s}, 1 \mathrm{H}, \mathrm{NH}) ; \mathrm{MS}(\mathrm{m} / \mathrm{z}): 342\left(\mathrm{M}^{+}\right)$; IR $\left(\mathrm{cm}^{-1}, \mathrm{KBr}\right): 3173(\mathrm{NH}), 2951(\mathrm{CH}), 1680(\mathrm{CO}), 713(\mathrm{C}-\mathrm{Cl})$; Anal calcd. for $\mathrm{C}_{18} \mathrm{H}_{15} \mathrm{ClN}_{2} \mathrm{O}_{3}: \mathrm{C}, 63.07 ; \mathrm{H}, 4.41 ; \mathrm{N}, 8.17$. Found: C, 62.97; H, 4.39; N, 8.19.

5-(4-Fluorobenzyl)-3-(4-chlorophenyl)-1,6-dihydro-6-pyridazinone (26): Yield: $57 \%$; m.p. $190{ }^{\circ} \mathrm{C}$; ${ }^{1} \mathrm{H}-\mathrm{NMR}\left(\mathrm{CDCl}_{3}, \delta\right.$, ppm): 3.61 (s, $2 \mathrm{H}$, $\left.\mathrm{CH}_{2}\right), 7.05(\mathrm{~s}, 1 \mathrm{H}, \mathrm{H}-4$, pyridazinone ring), 7.10 and 7.26 (d, each, $J=7.5 \mathrm{~Hz}$, $2 \mathrm{xA}_{2} \mathrm{~B}_{2}, p$-substituted benzyl ring), 7.41 and 7.60 (d, each, $J=7.8 \mathrm{~Hz}, 2 \mathrm{xA}_{2} \mathrm{~B}_{2}$, $p$-substituted phenyl ring), $11.14(\mathrm{~s}, 1 \mathrm{H}, \mathrm{NH}) ; \mathrm{MS}(\mathrm{m} / \mathrm{z}): 314\left(\mathrm{M}^{+}\right)$; IR $\left(\mathrm{cm}^{2}\right.$ 1, KBr): $3191(\mathrm{NH}), 2944(\mathrm{CH}), 1682(\mathrm{CO}), 719(\mathrm{C}-\mathrm{Cl})$; Anal calcd. for $\mathrm{C}_{17} \mathrm{H}_{12} \mathrm{ClFN}_{2} \mathrm{O}: \mathrm{C}, 64.87 ; \mathrm{H}, 3.84 ; \mathrm{N}, 8.90$. Found: $\mathrm{C}, 64.93 ; \mathrm{H}, 3.85 ; \mathrm{N}, 8.91$.

5-Benzyl-3-(4-methylphenyl)-1,6-dihydro-6-pyridazinone (27): Yield: $53 \%$; m.p. $168{ }^{\circ} \mathrm{C}$; ${ }^{1} \mathrm{H}-\mathrm{NMR}\left(\mathrm{CDCl}_{3}, \delta\right.$, ppm): $2.36\left(\mathrm{~s}, 3 \mathrm{H}, \mathrm{CH}_{3}\right), 3.59(\mathrm{~s}, 2 \mathrm{H}$, $\left.\mathrm{CH}_{2}\right), 6.86(\mathrm{~s}, 1 \mathrm{H}, \mathrm{H}-4$, pyridazinone ring), 7.02-7.43 ( $\mathrm{m}, 5 \mathrm{H}$, benzyl ring), 7.46 and 7.79 (d, each, $J=7.8 \mathrm{~Hz}, 2 \mathrm{xA}_{2} \mathrm{~B}_{2}, p$-substituted phenyl ring), $8.92(\mathrm{~s}, 1 \mathrm{H}$, $\mathrm{NH})$; MS $(\mathrm{m} / \mathrm{z}): 276\left(\mathrm{M}^{+}\right)$; IR $\left(\mathrm{cm}^{-1}, \mathrm{KBr}\right): 3185(\mathrm{NH}), 2952(\mathrm{CH}), 1676(\mathrm{CO})$; Anal calcd. for $\mathrm{C}_{18} \mathrm{H}_{16} \mathrm{~N}_{2} \mathrm{O}: \mathrm{C}, 78.24 ; \mathrm{H}, 5.84 ; \mathrm{N}, 10.14$. Found: $\mathrm{C}, 78.23 ; \mathrm{H}$, $5.87 ; \mathrm{N}, 10.17$.

5-(4-Chlorobenzyl)-3-(4-methylphenyl)-1,6-dihydro-6-pyridazinone (28): Yield: $48 \%$; m.p. $188^{\circ} \mathrm{C}$; ${ }^{1} \mathrm{H}-\mathrm{NMR}\left(\mathrm{CDCl}_{3}, \delta\right.$, ppm): 2.38 (s, $\left.3 \mathrm{H}, \mathrm{CH}_{3}\right), 3.94$ (s, $\left.2 \mathrm{H}, \mathrm{CH}_{2}\right), 7.24$ (s, 1H, $\mathrm{H}-4$, pyridazinone ring), 7.26 and 7.56 (d, each, $J=7.8$ $\mathrm{Hz}, 2 \mathrm{xA}_{2} \mathrm{~B}_{2}, p$-substituted benzyl ring), 7.28-7.35 (m, 4H, H-2,3,5,6, phenyl ring), $10.69(\mathrm{~s}, 1 \mathrm{H}, \mathrm{NH})$; $\mathrm{MS}(\mathrm{m} / \mathrm{z}): 310\left(\mathrm{M}^{+}\right) ; \mathrm{IR}\left(\mathrm{cm}^{-1}, \mathrm{KBr}\right): 3174(\mathrm{NH}), 2939$ (CH), $1683(\mathrm{CO}), 718(\mathrm{C}-\mathrm{Cl})$; Anal calcd. for $\mathrm{C}_{18} \mathrm{H}_{15} \mathrm{ClN}_{2} \mathrm{O}: \mathrm{C}, 69.57$; H, 4.86; N, 9.01. Found: C, 69.53; H, 4.87; N, 8.99.

5-(4-Nitrobenzyl)-3-(4-methylphenyl)-1,6-dihydro-6-pyridazinone (29): Yield: $47 \%$; m.p. $189^{\circ} \mathrm{C} ;{ }^{1} \mathrm{H}-\mathrm{NMR}\left(\mathrm{CDCl}_{3}, \delta\right.$, ppm): 2.37 (s, 3H, $\left.\mathrm{CH}_{3}\right), 3.41$ (s, $\left.2 \mathrm{H}, \mathrm{CH}_{2}\right), 7.13$ (s, $1 \mathrm{H}, \mathrm{H}-4$, pyridazinone ring), 7.31 and 8.01 (d, each, $J=8.1$ $\mathrm{Hz}, 2 \mathrm{xA}_{2} \mathrm{~B}_{2}, p$-substituted benzyl ring), 7.48 ( $\mathrm{m}, 2 \mathrm{H}, \mathrm{H}-3,5$, phenyl ring), 7.81 (m, 2H, H-2,4, phenyl ring), $11.13(\mathrm{~s}, 1 \mathrm{H}, \mathrm{NH}) ; \mathrm{MS}(\mathrm{m} / \mathrm{z}): 321\left(\mathrm{M}^{+}\right)$; IR $\left(\mathrm{cm}^{-}\right.$ 1, KBr): $3183(\mathrm{NH}), 2948(\mathrm{CH}), 1679(\mathrm{CO})$; Anal calcd. for $\mathrm{C}_{18} \mathrm{H}_{15} \mathrm{~N}_{3} \mathrm{O}_{3}: \mathrm{C}$, 67.28; H, 4.70; N, 13.08. Found: C, 67.23; H, 4.71; N, 13.07.

5-(4-Hydroxybenzyl)-3-(4-methylphenyl)-1,6-dihydro-6-pyridazinone (30): Yield: $47 \%$; m.p. $196{ }^{\circ} \mathrm{C} ;{ }^{1} \mathrm{H}-\mathrm{NMR}\left(\mathrm{CDCl}_{3}, \delta\right.$, ppm): 2.54 (s, $3 \mathrm{H}, \mathrm{CH}_{3}$ ), $3.43\left(\mathrm{~s}, 2 \mathrm{H}, \mathrm{CH}_{2}\right), 6.40(\mathrm{~s}, 1 \mathrm{H}, \mathrm{H}-4$, pyridazinone ring), $6.63(\mathrm{~m}, 2 \mathrm{H}, \mathrm{H}-2,6$, phenyl ring), $6.66(\mathrm{~m}, 2 \mathrm{H}, \mathrm{H}-2,6$, benzyl ring), $6.79(\mathrm{~m}, 2 \mathrm{H}, \mathrm{H}-3,5$, phenyl ring), $6.81\left(\mathrm{~m}, 2 \mathrm{H}, \mathrm{H}-3,5\right.$, benzyl ring), $12.25(\mathrm{~s}, 1 \mathrm{H}, \mathrm{NH}) ; \mathrm{MS}(\mathrm{m} / \mathrm{z}): 291\left(\mathrm{M}^{+}\right)$; IR ( $\left.\mathrm{cm}^{-1}, \mathrm{KBr}\right): 3173(\mathrm{NH}), 2957(\mathrm{CH}), 1685(\mathrm{CO})$; Anal calcd. for $\mathrm{C}_{18} \mathrm{H}_{16} \mathrm{~N}_{2} \mathrm{O}_{2}$ : C, 73.96; H, 5.52; N, 9.58. Found: C, 73.98; H, 5.51; N, 9.57.

5-(4-Methylbenzyl)-3-(4-methylphenyl)-1,6-dihydro-6-pyridazinone (31): Yield: $48 \%$; m.p. $182^{\circ} \mathrm{C} ;{ }^{1} \mathrm{H}-\mathrm{NMR}\left(\mathrm{CDCl}_{3}, \delta\right.$, ppm): 2.29 and 2.31 (s, each, $6 \mathrm{H}$, $\left.2 \mathrm{xCH}_{3}\right), 3.67\left(\mathrm{~s}, 2 \mathrm{H}, \mathrm{CH}_{2}\right), 6.51$ (s, $1 \mathrm{H}, \mathrm{H}-4$, pyridazinone ring), 7.31 and 7.85 (d, each, $J=7.8 \mathrm{~Hz}, 2 \mathrm{xA}_{2} \mathrm{~B}_{2}, p$-substituted benzyl ring), 7.37 (m, $2 \mathrm{H}, \mathrm{H}-3,5$, phenyl ring), 7.59 (m, 2H, H-2,6, phenyl ring), 10.51 (s, 1H, NH); MS ( $m / z)$ : 289 $\left(\mathrm{M}^{+}\right)$; IR $\left(\mathrm{cm}^{-1}, \mathrm{KBr}\right): 3182(\mathrm{NH}), 2940(\mathrm{CH}), 1676(\mathrm{CO})$; Anal calcd. for $\mathrm{C}_{19} \mathrm{H}_{18} \mathrm{~N}_{2} \mathrm{O}: \mathrm{C}, 78.59 ; \mathrm{H}, 6.25 ; \mathrm{N}, 9.65$. Found: C, 78.53; H, 6.27; N, 9.67.

5-(4-Methoxybenzyl)-3-(4-methylphenyl)-1,6-dihydro-6-pyridazinone (32): Yield: $43 \%$; m.p. $192{ }^{\circ} \mathrm{C}$; ${ }^{1} \mathrm{H}-\mathrm{NMR}\left(\mathrm{CDCl}_{3}, \delta\right.$, ppm): 2.37 (s, $\left.3 \mathrm{H}, \mathrm{CH}_{3}\right)$, $3.67\left(\mathrm{~s}, 3 \mathrm{H}, \mathrm{OCH}_{3}\right), 3.97\left(\mathrm{~s}, 2 \mathrm{H}, \mathrm{CH}_{2}\right), 6.79(\mathrm{~s}, 1 \mathrm{H}, \mathrm{H}-4$, pyridazinone ring), 7.37 and 7.82 (d, each, $J=8.1 \mathrm{~Hz}, 2 \times \mathrm{A}_{2} \mathrm{~B}_{2}, p$-substituted benzyl ring), $7.49(\mathrm{~m}$, $2 \mathrm{H}, \mathrm{H}-3,5$, phenyl ring), 7.72 (m, 2H, H-2,6, phenyl ring), 11.15 (s, $1 \mathrm{H}, \mathrm{NH})$; MS $(\mathrm{m} / \mathrm{z}): 305\left(\mathrm{M}^{+}\right)$; IR $\left(\mathrm{cm}^{-1}, \mathrm{KBr}\right): 3186(\mathrm{NH}), 2944(\mathrm{CH}), 1682(\mathrm{CO})$; Anal calcd. for $\mathrm{C}_{19} \mathrm{H}_{18} \mathrm{~N}_{2} \mathrm{O}_{2}: \mathrm{C}, 74.49 ; \mathrm{H}, 5.92 ; \mathrm{N}, 9.14$. Found: C, 74.53; H, 5.91; N, 9.13 .

5-(4-Hydroxy-3-methoxy-benzyl)-3-(4-methylphenyl)-1,6-dihydro-6pyridazinone (33): Yield: $51 \%$; m.p. $180{ }^{\circ} \mathrm{C}$; ${ }^{1} \mathrm{H}-\mathrm{NMR}\left(\mathrm{CDCl}_{3}, \delta, \mathrm{ppm}\right)$ :) $\delta$ $2.39\left(\mathrm{~s}, 3 \mathrm{H}, \mathrm{CH}_{3}\right), 3.25\left(\mathrm{~s}, 3 \mathrm{H}, \mathrm{OCH}_{3}\right), 3.92\left(\mathrm{~s}, 2 \mathrm{H}, \mathrm{CH}_{2}\right), 6.82(\mathrm{~s}, 1 \mathrm{H}, \mathrm{H}-4$, pyridazinone ring), 7.04 (m, 1H, H-6, benzyl ring), 7.25 (m, 2H, H-3,5, phenyl ring), $7.53(\mathrm{~m}, 1 \mathrm{H}, \mathrm{H}-2$, benzyl ring), $7.68(\mathrm{~m}, 2 \mathrm{H}, \mathrm{H}-2,6$, phenyl ring), 7.75 $\left(\mathrm{m}, 1 \mathrm{H}, \mathrm{H}-5\right.$, benzyl ring), $10.73(\mathrm{~s}, 1 \mathrm{H}, \mathrm{NH}) ; \mathrm{MS}(\mathrm{m} / \mathrm{z}): 321\left(\mathrm{M}^{+}\right)$; IR $\left(\mathrm{cm}^{-}\right.$ 1, $\mathrm{KBr}): 3189(\mathrm{NH}), 2952(\mathrm{CH}), 1687(\mathrm{CO})$; Anal calcd. for $\mathrm{C}_{19} \mathrm{H}_{18} \mathrm{~N}_{2} \mathrm{O}_{3}: \mathrm{C}$, $70.79 ; \mathrm{H}, 5.63 ; \mathrm{N}, 8.69$. Found: C, 70.83; H, 5.65; N, 8.67.

5-(4-Fluorobenzyl)-3-(4-methylphenyl)-1,6-dihydro-6-pyridazinone (34): Yield: $49 \%$; m.p. $174^{\circ} \mathrm{C}$; ${ }^{1} \mathrm{H}-\mathrm{NMR}\left(\mathrm{CDCl}_{3}, \delta\right.$, ppm): $2.36\left(\mathrm{~s}, 3 \mathrm{H}, \mathrm{CH}_{3}\right), 3.95$ (s, $\left.2 \mathrm{H}, \mathrm{CH}_{2}\right), 7.01$ (s, $1 \mathrm{H}, \mathrm{H}-4$, pyridazinone ring), 7.05 and 7.55 (d, each, $J=7.5$ $\mathrm{Hz}, 2 \mathrm{xA}_{2} \mathrm{~B}_{2}, p$-substituted benzyl ring), 7.21-7.29 (m, 4H, H-2,3,6,5, phenyl ring), $11.42(\mathrm{~s}, 1 \mathrm{H}, \mathrm{NH})$; $\mathrm{MS}(\mathrm{m} / \mathrm{z}): 293\left(\mathrm{M}^{+}\right)$. IR $\left(\mathrm{cm}^{-1}, \mathrm{KBr}\right): 3183(\mathrm{NH})$, $2948(\mathrm{CH}), 1672(\mathrm{CO})$; Anal calcd. for $\mathrm{C}_{18} \mathrm{H}_{15} \mathrm{FN}_{2} \mathrm{O}$ : C, 73.45; H, 5.14; N, 9.52. Found: C, 73.43; H, 5.17; N, 9.53.

Antitubercular activity $[15,16]$

The antitubercular screening was carried out against Mycobacterium tuberculosis $\mathrm{H}_{37} \mathrm{Rv}$ (ATCC 27294) in Middle brook 7H11 agar medium with OADC (oleic acid albumin dextrose catalase) growth supplement. 10 fold serial dilutions of each test compound/drug (in DMSO/Water mixture) were incorporated into the agar medium. Inoculum of M. tuberculosis $\mathrm{H}_{37} \mathrm{Rv}$ were prepared from fresh Middlebrook 7H11 agar slants with OADC growth supplement adjusted to $1 \mathrm{mg} / \mathrm{mL}$ (wet weight) in Tween $80(0.05 \%)$ saline diluted to $10^{-2}$ to give a concentration of approximately $10^{7} \mathrm{cfu} / \mathrm{mL}$. A $5 \mu \mathrm{L}$ amount of bacterial suspension was spotted into $7 \mathrm{H} 11$ agar tubes containing 10 -fold serial dilutions of drugs per $\mathrm{mL}$. The tubes were incubated at $37^{\circ} \mathrm{C}$, and final readings were recorded after 30 days. The minimum inhibitory concentration (MIC) is defined as the minimum concentration of compound required to give complete inhibition of bacterial growth. The $M I C$ of the standard drug streptomycin was $10 \mu \mathrm{g} / \mathrm{mL}$. The results of the pharmacological evaluation have been listed in Table $\mathbf{1}$.

\section{RESULT AND DISCUSSION}

\section{Chemistry}

$2(3 H)$-Furanones (3-18) on reaction with hydrazine hydrate in $n$-propanol gave 16 title compounds i.e. 5-(substituted benzyl)-3-aryl-1,6-dihydro-6pyridazinone derivatives (19-34). 2(3H)-Furanones (3-18) were prepared using 3-(4-substituted benzoyl)propionic acid (1-2) following the previously reported methods of modified Perkin's reaction in higher yields [14]. The 3-(4-substituted benzoyl)propionic acid $(\mathbf{1}, \mathbf{2})$ was synthesized according to Friedel Craft's acylation reaction condition using chlorobenzene or toluene (Scheme-1).<smiles>[R]c1ccccc1</smiles><smiles>[R]c1ccc(C(=O)CCC(=O)O)cc1</smiles><smiles>[R]Cc1cccc(/C=C2\C=C(c3ccccc3)OC2=O)c1</smiles>

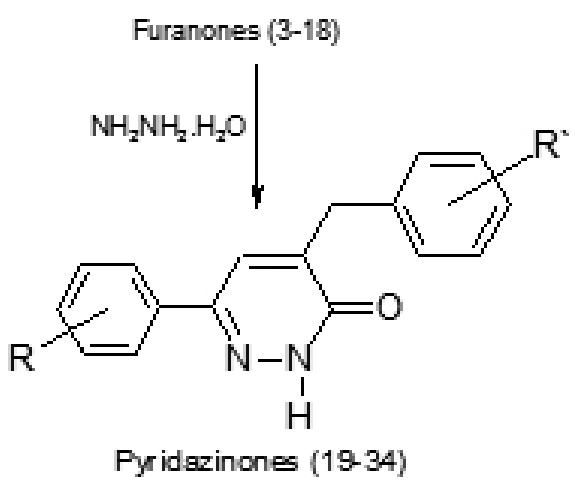

Scheme 1: Protocol for synthesis of pyridazinones. 
Antitubercular evaluation

The antitubercular screening was carried out against Mycobacterium tuberculosis $\mathrm{H}_{37} \mathrm{Rv}$ (ATCC 27294) (Table 1). The results illustrated that 5-(4-hydroxy-3-methoxybenzyl)-3-(4-chlorophenyl)-1,6-dihydro-6pyridazinone (25) showed best antitubercular activity among the synthesized compounds with $M I C-12.5 \mu \mathrm{g} / \mathrm{mL}$. Four compounds, 5-(4-nitrobenzyl)-3(4-chloro-phenyl)-1,6-dihydro-6-pyridazinone (21), 5-(4-hydroxybenzyl)3-(4-chlorophenyl)-1,6-dihydro-6-pyridazinone (22), 5-(4-nitrobenzyl)-3(4-methylphenyl)-1,6-dihydro-6-pyridazinone (29) and 5-(4-hydroxy-3methoxybenzyl)-3-(4-methylphenyl)-1,6-dihydro-6-pyridazinone (33) were also significant in their antitubercular action with $M I C-25 \mu \mathrm{g} / \mathrm{mL}$. Rests of the compounds showed $M I C$-values of $50 \mu \mathrm{g} / \mathrm{mL}$. Pyridazinones derived from 4-chloro-furanones were found to have better activity than those derived from 4-methyl-furanones. Disubstituted phenyl rings $(\mathbf{2 5} \& \mathbf{3 3})$ at $5^{\text {th }}$ position of pyridazinone ring showed better antitubercular activity than unsubstituted or mono-substituted phenyl rings. Among the mono-substituted phenyl rings at $5^{\text {th }}$ position of pyridazinone ring, presence of nitro group ( $\left.21 \& 29\right)$ showed significant antitubercular activity. (Table 1).

Table 1: Antitubercular activity of the Pyridazinone derivatives 19-34.

\begin{tabular}{|c|c|c|c|}
\hline Compound & $\mathbf{R}$ & $\mathbf{R}^{\prime}$ & $\boldsymbol{M I C}$ values $(\boldsymbol{\mu g} / \mathbf{m L})$ \\
\hline 19 & $4-\mathrm{Cl}$ & $\mathrm{H}$ & 50 \\
\hline 20 & $4-\mathrm{Cl}$ & $4-\mathrm{Cl}$ & 50 \\
\hline 21 & $4-\mathrm{Cl}$ & $4-\mathrm{NO}_{2}$ & 25 \\
\hline 22 & $4-\mathrm{Cl}$ & $4-\mathrm{OH}$ & 25 \\
\hline 23 & $4-\mathrm{Cl}$ & $4-\mathrm{CH}_{3}$ & 100 \\
\hline 24 & $4-\mathrm{Cl}$ & $4-\mathrm{OCH}_{3}$ & 50 \\
\hline 25 & $4-\mathrm{Cl}$ & $4-\mathrm{OH} ; 3-\mathrm{OCH}_{3}$ & 12.5 \\
\hline 26 & $4-\mathrm{Cl}$ & $4-\mathrm{F}$ & 50 \\
\hline 27 & $4-\mathrm{CH}_{3}$ & $\mathrm{H}$ & 50 \\
\hline 28 & $4-\mathrm{CH}_{3}$ & $4-\mathrm{Cl}_{3}$ & 50 \\
\hline 29 & $4-\mathrm{CH}_{3}$ & $4-\mathrm{NO}_{2}$ & 25 \\
\hline 30 & $4-\mathrm{CH}_{3}$ & $4-\mathrm{OH}_{3}$ & 50 \\
\hline 31 & $4-\mathrm{CH}_{3}$ & $4-\mathrm{CH}_{3}$ & 50 \\
\hline 32 & $4-\mathrm{CH}_{3}$ & $4-\mathrm{OCH}$ & 50 \\
\hline 33 & $4-\mathrm{CH}_{3}$ & $4-\mathrm{OH} ; 3-\mathrm{OCH}_{3}$ & 25 \\
\hline 34 & $4-\mathrm{CH}_{3}$ & $4-\mathrm{F}$ & 50 \\
\hline Streptomycin & - & - & 10 \\
\hline
\end{tabular}

\section{CONCLUSIONS} 25, 5-(4-hydroxy-3-methoxybenzyl)-3-(4-chlorophenyl)-1,6-dihydro-6-

To sum up, among the synthesized 16 newer pyridazinones, compound pyridazinone emerged as lead compound with good antitubercular activity. The study showed the antitubercular potential of pyridazinones.

Acknowledgements: The authors are thankful to UGC for financial assistance under major-research project scheme. We are thankful to Prof MSY Khan, Professor Emeritus, Jamia Hamdard, New Delhi for his valuable suggestions.

\section{REFERENCES}

1.- M. Sonmez, I. Berber, E. Akbas, Eur. J. Med. Chem. 41, 101, (2006).

2.- S. A. Abubshait, Molecules 12, 25, (2007).

3.- R. Rossotti, S. Rusconi, HIV Therapy 3, 63, (2009)

4.- S. Ahmad, I. G. Rathish, S. Bano, M. S. Alam, K. Javed, J. Enzy Inh. Med. Chem. 25,266, (2010).

5.- D. S. Dogruer, M. F. Sahin, S. Unlu, S. Ito, Arch Pharm (Weinheim) 333, 79, (2000)

6.- D. Dogruer, M. F. Sahin, Turk. J. Chem. 27, 727, (2003).

7. R. Sivakumar, N. Anbalagan, V. Gunasekaran, J. T. Leonard, Biol. Pharm. Bull. 26, 1407, (2003).

8.- T. Costas, P. Besada, A. Piras, L. Acevedo, M. Yanez, F. Orallo, R. Laguna, C. Teran, Bioorg Med Chem Lett. 20, 6624, (2010).

9.- A. Okushima, A. Narimatsu, M. Kobayashi, R. Furuya, K. Tsuda, Y. Kitada, J. Med. Chem. 30, 1157, (1987).

10.- T. Yamada, Y. Nobuhara, H. Shimamura, K. Yoshihara, A. Yamaguchi, M. Ohki, Chem. Pharm. Bull. 29, 3433, (1981).

11.- B. R. Bloom, C. J. L. Murray, Science 257, 1055, (1992).

12.- P. F. Barnes, A. B. Blotch, P. T. Davidson, D. E. Snider, N. Engl. J. Med. 324, 1644, (1991).

13.- M. A. Ali, M. S. Yar, A. A. Siddiqui, A. Husain, M. Abdullah, Acta Polo. Pharm-Drug Research 63, 435, (2007).

14.- A. Husain, M. M. Alam, S. M. Hasan, M. S. Yar, Acta Polo. Pharm-Drug Research 66, 173, (2009).

15.- W. K. Elmer, D. A. Stephen, M. J. William, C. S. Paul, C. W. Washing, Text Book of Diagnostic Microbiology, $5^{\text {th }}$ ed, Lippincot Publishers, 2002.

16.- J. B. Ellen, R. P. Lancer, M. F. Sydney, Bailey and Scott's Diagnostic Microbiology, $9^{\text {th }}$ ed, 2000. 